\title{
A prospective observational study of the prevalence and risk factors for colonization by antibiotic resistant bacteria in patients at admission to hospital in Singapore
}

Barnaby E Young ${ }^{1 *}$, David C Lye ${ }^{1,2}$, Prabha Krishnan³ , Siew Pang Chan ${ }^{1,4}$ and Yee Sin Leo ${ }^{1,2}$

\begin{abstract}
Background: Drug resistant organisms pose an increasing threat to the successful treatment of common infections. Understanding colonization patterns of these bacteria is important for effective antibiotic treatment and infection control guidelines.

Methods: A prospective observational study was performed to determine the prevalence of colonization with extended-spectrum $\beta$-lactamase-producing Enterobacteriaceae (ESBL-E), methicillin-resistant Staphylococcus aureus (MRSA), and vancomycin-resistant Enterococcus (VRE) among patients admitted via the emergency department to a public tertiary hospital in Singapore. Anterior nares, groin, axillary and rectal swabs were collected at admission and cultured using standard bacteriological techniques. Clinical data including healthcare contact within the past 12 months and recent antibiotic use was collected and analyzed using a logistic regression model.

Results: 1006 patients were screened. 124 (12.4\%) were colonized by ESBL-E, 18 (1.8\%) by MRSA while no VRE was detected. Antibiotic use within the past month was the only significant predictor for ESBL-E colonization in the regression model, with an adjusted odds ratio (AOR) of 2.58 (1.04 to 6.42). In participants recently prescribed antibiotics and hospitalized in the previous 3 months, 29.4\% were colonized by ESBL-E. This represented 20.2\% of the total ESBL-E burden, and ESBL-E was also detected in $6.3 \%$ of participants with no healthcare contact. Hospitalization and outpatient hospital visits predicted MRSA colonization in the univariate analysis. Neither was statistically significant in the logistic regression model, with AORs for MRSA colonization following hospitalization in the past 3 and 12 months of 3.81 [95\% Cl 0.84-17.28] and 3.48 [0.64-18.92] respectively.
\end{abstract}

Conclusion: A high prevalence of colonization with ESBL-E was evident among patients at admission, even in the absence of recent antibiotic use or contact with healthcare.

Keywords: Antibiotics, Colonization, ESBL, Fecal carriage, MRSA

\section{Background}

Methicillin-resistant Staphylococcus aureus (MRSA) and vancomycin-resistant Enterococcus (VRE) emerged in continental Asia following dissemination from Europe and North America [1]. Over the past decade these pathways have reversed, with Asia the probable source

\footnotetext{
* Correspondence: Barnaby_young@ttsh.com.sg

'Institute of Infectious Diseases and Epidemiology, Communicable Diseases Centre, Singapore, Singapore

Full list of author information is available at the end of the article
}

of broad-spectrum $\beta$-lactamases such as CTX-M-15 and NDM-1 [2,3]. Both are now identified globally.

VRE is relatively uncommonly identified in Asia, while MRSA has become established in Asian hospitals and is emerging in the community [4]. Extended-spectrum $\beta$-lactamase-producing Enterobacteriaceae (ESBL-E) have reached an extraordinary prevalence in clinical isolates from both hospitals and the community. Surveillance by the Study for Monitoring Antimicrobial Trends (SMART) in the Asia-Pacific region from 2008-2010 detected ESBL-E in $32.8 \%$ of urinary tract infections 
and $11.2 \%$ of intra-abdominal infections (IAI) [5,6]. Almost 20\% of ESBL-E were cultured from communityonset IAI. ESBL-E fecal carriage rates among healthy volunteers in Asia have ranged from $6.4 \%$ in Japan to an extraordinary $61.7 \%$ in rural Thailand $[7,8]$.

As many bacterial infections derive from host flora, colonization by drug resistant organisms is a risk for drug resistant infections $[9,10]$. An increased mortality with these infections correlates with delayed recognition and inadequate empirical therapy $[11,12]$. Knowledge of local resistance patterns is therefore important for evidence-based institutional empiric antibiotic treatment and infection control guidelines. We developed a prospective study to determine the prevalence of colonization by ESBL-E, MRSA and VRE in patients admitted to our hospital in Singapore, and to explore some of the associated risk factors.

\section{Methods}

\section{Participants and setting}

We conducted this prospective observational study at the emergency department (ED) of Tan Tock Seng Hospital, Singapore. This is a public tertiary hospital of more than 1500 inpatient beds and a busy ED with more than 11,000 attendances and almost 4000 admissions per month in 2006.

Consecutive patients over 16 years of age planned for hospital admission were approached to participate. The recruitment period was $12-8$ pm Monday to Friday from November 2006 to February 2007. Pregnant women were excluded. Target recruitment was 1000, calculated to estimate the colonization rate for MRSA with $95 \%$ confidence within $1 \%$ of its actual value, based on an expected value of $2 \%$ from previous institutional surveillance studies.

Anterior nares, groin, axilla and rectum were swabbed by a research nurse in the ED. If the participant declined or was unsuitable for rectal swabbing, freshly passed stools were collected within 24 hours of admission. Clinical data recorded at admission from the participant or next-of-kin included age, gender, co-morbidities, residence in a care facility, hospitalization or outpatient (hospital and primary care) healthcare contact within the last 12 months and receipt of antibiotics in the last 3 months.

Written informed consent was obtained from all participants or the next-of-kin and the study was approved by the Institutional Ethics Committee (the National Healthcare Group Domain Specific Review Board).

\section{Bacteria identification and resistance testing}

Rectal swabs/stool specimens were inoculated onto two MacConkey agar plates supplemented with $1 \mathrm{mg} / \mathrm{L}$ of either cefotaxime or ceftazidime and incubated for 48 hours.
Enterobacteriaceae were identified by Microbact $12 \mathrm{~A}$ (Oxoid, UK) and the presence of ESBL screened with double-disc synergy using cefotaxime $30 \mu \mathrm{g}$ and ceftazidime $30 \mu \mathrm{g}$ discs placed $25 \mathrm{~mm}$ from an amoxicillinclavulanate $(20 \mu \mathrm{g}-10 \mu \mathrm{g})$ disc. Isolates screening positive for ESBL were tested with a multiplex PCR assay for detection of CTX-M clusters, according to published protocols [13].

Nares, groin and axillary swabs were inoculated onto mannitol salt agar supplemented with oxacillin $6 \mu \mathrm{g} / \mathrm{ml}$ and incubated for 48 hours. MRSA was identified by gram stain, tube coagulase test and antimicrobial susceptibility testing.

Rectal swabs/stool specimens were also inoculated onto bile-esculin-azide agar containing $6 \mathrm{mgs} / \mathrm{L}$ vancomycin for 48 hours and black colonies identified as enterococci by conventional methods including motility and pigment production. Vancomycin resistance was confirmed using vancomycin minimum concentration (MIC) E-test (AB BioDisk, Sweden) and an in-house van gene polymerase chain reaction (PCR).

Disc diffusion anti-microbial susceptibility testing was performed for all isolates in accordance with the 2007 Clinical Laboratory Standard Institute Guidelines [14].

\section{Data analysis}

An initial exploratory analysis was performed with Fisher's exact test. Logistic regression was used to determine which covariates were significantly associated with the detection of ESBL-E, MRSA or VRE. All identified covariates were entered into the model before employing a backward elimination algorithm. Goodness-of-fit was estimated with the Hosmer Lemeshow (H-L) test. The sensitivity and specificity of individual measures for predicting colonization was estimated using the efficient-score method.

Participants who reported no contact with healthcare, healthcare workers, antibiotics or nursing homes were used as a surrogate for the community prevalence of colonization by ESBL-E, MRSA or VRE.

Statistical analyses were performed using Stata 12.0 (Stata Corporation, U.S.A.). All statistical tests were conducted at a two-sided 5\% level of significance.

\section{Results}

1006 out of 1592 patients (63.2\%) admitted during the recruitment period consented to participate. Rectal swabs were obtained from 1001 (99.5\%); 3 (0.3\%) provided stool specimens; $2(0.2 \%)$ did not provide any sample. MRSA screening was performed on all 1006.

Baseline characteristics and findings are summarized in Table 1. 124 (12.4\%) were colonized by ESBL-E, 18 $(1.8 \%)$ by MRSA, and no VRE was detected. 
Table 1 Sample characteristics and univariate analysis

\begin{tabular}{|c|c|c|c|c|c|c|}
\hline & MRSA No $(n=988)$ & Yes $(n=18)$ & p-value & ESBL-E No $(n=880)$ & Yes $(n=124)$ & $\mathrm{p}$-value \\
\hline \multicolumn{7}{|l|}{ Demographics: } \\
\hline \multicolumn{7}{|l|}{ Gender } \\
\hline Male & $643(98.2 \%)$ & $12(1.8 \%)$ & NS & $571(87.2 \%)$ & $84(12.8 \%)$ & NS \\
\hline Female & $345(98.3 \%)$ & $6(1.7 \%)$ & & $309(88.5 \%)$ & $40(11.5 \%)$ & \\
\hline \multicolumn{7}{|l|}{ Ethnicity } \\
\hline Chinese & $639(97.6 \%)$ & $16(2.4 \%)$ & NS & $571(87.2 \%)$ & $84(12.8 \%)$ & NS \\
\hline Malay & $166(99.4 \%)$ & $1(0.6 \%)$ & & 149 (89.2\%) & $18(10.8 \%)$ & \\
\hline Indian & $133(99.2 \%)$ & $1(0.8 \%)$ & & $115(86.5 \%)$ & $18(13.5 \%)$ & \\
\hline Others & $50(100.0 \%)$ & $0(0.0 \%)$ & & $45(91.8 \%)$ & $4(8.2 \%)$ & \\
\hline \multicolumn{7}{|c|}{ Residence in Nursing Home } \\
\hline No & $966(98.2 \%)$ & $18(1.8 \%)$ & NS & $860(87.6 \%)$ & $122(12.4 \%)$ & NS \\
\hline Yes & $22(100.0 \%)$ & $0(0.0 \%)$ & & $20(90.9 \%)$ & $2(9.1 \%)$ & \\
\hline \multicolumn{7}{|c|}{ Comorbid conditions: } \\
\hline \multicolumn{7}{|l|}{ Diabetes Mellitus } \\
\hline No & $782(98.7 \%)$ & $10(1.3 \%)$ & 0.035 & $697(88.2 \%)$ & $93(11.8 \%)$ & NS \\
\hline Yes & $206(96.3 \%)$ & $8(3.7 \%)$ & & $183(85.5 \%)$ & $31(11.5 \%)$ & \\
\hline \multicolumn{7}{|l|}{ ESRF } \\
\hline No & 977 (98.2\%) & $18(1.8 \%)$ & NS & $869(87.5 \%)$ & $124(12.5 \%)$ & NS \\
\hline Yes & $11(100.0 \%)$ & $0(0.0 \%)$ & & $11(100.0 \%)$ & $0(0.0 \%)$ & \\
\hline \multicolumn{7}{|l|}{ Liver Cirrhosis } \\
\hline No & 977 (98.2\%) & $18(1.8 \%)$ & NS & $869(87.5 \%)$ & $124(12.5 \%)$ & NS \\
\hline Yes & 11 (100.0\%) & $0(0.0 \%)$ & & $11(100.0 \%)$ & $0(0.0 \%)$ & \\
\hline \multicolumn{7}{|l|}{ Cancer } \\
\hline No & $962(98.2 \%)$ & $18(1.8 \%)$ & NS & $854(87.3 \%)$ & 124 (12.7\%) & NS \\
\hline Yes & $26(100.0 \%)$ & $0(100.0 \%)$ & & $26(100.0 \%)$ & $0(0.0 \%)$ & \\
\hline \multicolumn{7}{|l|}{ HIV } \\
\hline No & 985 (98.2\%) & $18(1.8 \%)$ & NS & $878(87.7 \%)$ & $123(12.3 \%)$ & NS \\
\hline Yes & $3(100.0 \%)$ & $0(0.0 \%)$ & & $2(66.7 \%)$ & $1(33.3 \%)$ & \\
\hline \multicolumn{7}{|l|}{ Corticosteroids } \\
\hline No & $944(98.2 \%)$ & $17(1.8 \%)$ & NS & $845(88.1 \%)$ & $114(11.9 \%)$ & NS \\
\hline Yes & $43(97.7 \%)$ & $1(2.3 \%)$ & & $34(77.3 \%)$ & $10(22.7 \%)$ & \\
\hline \multicolumn{7}{|c|}{ Healthcare risk factors: } \\
\hline \multicolumn{7}{|l|}{ Hospitalization } \\
\hline Within 3 month & 155 (93.4\%) & $11(6.6 \%)$ & $<0.001$ & $131(78.9 \%)$ & $35(21.1 \%)$ & $<0.001$ \\
\hline Within 12 month & $297(95.5 \%)$ & $14(4.5 \%)$ & $<0.001$ & $257(82.6 \%)$ & $54(17.4 \%)$ & 0.002 \\
\hline \multicolumn{7}{|l|}{ Oral antibiotics } \\
\hline Within 1 month & 279 (97.2\%) & $8(2.8 \%)$ & NS & 227 (79.1\%) & $60(20.9 \%)$ & $<0.001$ \\
\hline Within 3 month & $343(97.4 \%)$ & $9(2.6 \%)$ & NS & $286(81.3 \%)$ & $66(18.7 \%)$ & $<0.001$ \\
\hline \multicolumn{7}{|c|}{ Outpatient hospital visit } \\
\hline Within 3 month & 402 (96.9\%) & $13(3.1 \%)$ & 0.013 & $347(83.8 \%)$ & $67(16.2 \%)$ & 0.002 \\
\hline Within 12 month & $536(97.3 \%)$ & $15(2.7 \%)$ & 0.016 & $470(85.4 \%)$ & $80(14.6 \%)$ & 0.021 \\
\hline \multicolumn{7}{|l|}{ Primary care visit } \\
\hline Within 3 month & $657(97.6 \%)$ & $16(2.4 \%)$ & 0.046 & $584(87.0 \%)$ & 87 (13.0\%) & NS \\
\hline
\end{tabular}


Table 1 Sample characteristics and univariate analysis (Continued)

\begin{tabular}{|c|c|c|c|c|c|c|}
\hline Within 12 month & 729 (97.9\%) & $16(2.1 \%)$ & NS & $652(87.8 \%)$ & 91 (12.2\%) & NS \\
\hline \multicolumn{7}{|l|}{ HCW contact } \\
\hline No & 853 (98.3\%) & 15 (1.7\%) & NS & 759 (87.5\%) & $108(12.5 \%)$ & NS \\
\hline Yes & 135 (97.8\%) & $3(2.2 \%)$ & & $121(88.3 \%)$ & 16 (11.7\%) & \\
\hline
\end{tabular}

ESRF: End-stage Renal Failure; HIV: Human Immunodeficiency Virus; HCW: Healthcare Worker.

\section{Univariate analysis and logistic regression}

Inpatient or outpatient hospital care and recent antibiotic prescription were significant risk factors for ESBLE colonization in the univariate analysis (Table 1). In the logit model only antibiotic use within 1 month prior to admission significantly predicted ESBL-E colonization, with an AOR of 2.58 [1.04 to 6.42] (Table 2).

For MRSA colonization, inpatient or outpatient hospital care within the previous 3 or 12 months were significantly associated in the univariate analysis (Table 1). Neither factor was statistically significant in the logit model. Adjusted odds ratios (AOR) for MRSA colonization following hospitalization in the past 3 and 12 months were 3.81 [95\% CI 0.84-17.28] and 3.48 [0.64-18.92] respectively (Table 2).

Both logit models provided a satisfactory fit to the data $(\mathrm{H}-\mathrm{L}$ test: $\mathrm{ESBL}-\mathrm{E} \mathrm{p}$-value $=0.52, \mathrm{MRSA} \mathrm{p}$-value $=0.87)$.

\section{Community colonization}

95 participants reported no contact with healthcare, healthcare workers, antibiotics or nursing homes; 6 (6.3\%) were colonized by ESBL-E, 0 by MRSA and 0 by VRE.

The importance of recent antibiotic use for ESBL-E colonization was evident from a comparison of colonization rates in participants stratified by attendance at different levels of the healthcare system in the previous three months, and antibiotic use in the previous month (Table 3). Baseline colonization prevalence in each subgroup was not significantly different from the putative community prevalence of $6.3 \%$ (Fisher's exact test), however prevalence was significantly higher than baseline if antibiotics were administered in the previous month. In participants who had been recently admitted to hospital and received antibiotics, 29.4\% were colonized by ESBL-E.

\section{Predicting colonization}

Oral antibiotics in the past month predicted ESBL-E fecal carriage with a sensitivity of $48.4 \%$ [95\% CI 39.4-57.5\%] and specificity of 74.2\% [71.2-77.0\%]. Specificity could be improved by filtering for additional hospitalization in the past 3 months, but with a significant loss of sensitivity: this was $22.1 \%$ [15.3-28.2\%] sensitive and $89.4 \%$ [87.0-91.3\%] specific. Specificity was improved further by combining oral antibiotics in the past month and hospitalization within the past 3 months. This predicted ESBL-E fecal carriage with 20.2\% [13.7-28.5\%] sensitivity and 93.2\% [91.3-94.7\%] specificity.

Hospitalization within the last 3 months was $61.1 \%$ [95\% CI 36.1-81.7\%] sensitive and 84\% [81.9-86.5\%] specific for MRSA colonization. Sensitivity could be improved to $77.8 \%$ [51.9-92.6\%], but with a specificity of

Table 2 Multiple logistic regression analyses of MRSA and ESBL-E colonization risk factors

\begin{tabular}{|c|c|c|c|c|}
\hline Featured covariates* & MRSA AOR & 95\% C.I. & ESBL-E AOR & 95\% C.I. \\
\hline \multicolumn{5}{|l|}{ Hospitalization } \\
\hline Within 3 month & 3.81 & 0.84 to 17.28 & 1.38 & 0.71 to 2.69 \\
\hline Within 12 month & 3.48 & 0.64 to 18.92 & 1.35 & 0.73 to 2.48 \\
\hline \multicolumn{5}{|l|}{ Use of oral antibiotics } \\
\hline Within 1 month & 1.57 & 0.17 to 14.43 & $2.58^{* *}$ & 1.04 to 6.42 \\
\hline Within 3 month & 0.67 & 0.08 to 6.78 & 0.94 & 0.38 to 2.36 \\
\hline \multicolumn{5}{|l|}{ Outpatient visit } \\
\hline Within 3 month & 0.97 & 0.17 to 5.64 & 1.36 & 0.69 to 2.69 \\
\hline Within 12 month & 1.43 & 0.18 to 11.13 & 0.89 & 0.43 to 1.80 \\
\hline \multicolumn{5}{|l|}{ Primary care visit } \\
\hline Within 3 month & Not estimated & Not estimated & 2.08 & 0.72 to 6.01 \\
\hline Within 12 month & Not estimated & Not estimated & 0.41 & 0.14 to 1.24 \\
\hline Contact with Healthcare Worker & 1.30 & 0.32 to 5.33 & 0.86 & 0.47 to 1.57 \\
\hline
\end{tabular}

*The models are adjusted for demographics and co-morbid conditions. **Statistically significant at $5 \%$. 


\begin{tabular}{|c|c|c|c|c|c|}
\hline \multirow[t]{2}{*}{ Sub-group } & \multicolumn{2}{|c|}{ Number of participants } & \multirow[t]{2}{*}{ Prevalence } & \multirow{2}{*}{$\begin{array}{l}\text { Odds ratio } \\
\text { (95\% C.I.) }\end{array}$} & \multirow[t]{2}{*}{ p-value } \\
\hline & ESBL-E colonized & Not colonized & & & \\
\hline No healthcare contact or antibiotics & 6 & 95 & $6.3 \%$ & & \\
\hline Primary care, no antibiotics & 36 & 452 & $8.0 \%$ & $2.90^{*}(1.84$ to 4.57$)$ & $<0.0001$ \\
\hline Primary care and antibiotics & 51 & 221 & $23.1 \%$ & & \\
\hline Outpatient, no antibiotics & 24 & 245 & $9.8 \%$ & $1.78^{*}$ (1.05 to 3.02$)$ & 0.033 \\
\hline Outpatient and antibiotics & 43 & 247 & $17.4 \%$ & & \\
\hline Hospitalization, no antibiotics & 10 & 81 & $12.3 \%$ & $2.38^{*}(1.08$ to 5.02$)$ & 0.032 \\
\hline Hospitalization and antibiotics & 25 & 85 & $29.4 \%$ & & \\
\hline
\end{tabular}

*Statistically significant at $5 \%$.

$69 \%$ [70.0-72.8\%] using hospitalization in the past 12 months to predict MRSA colonization.

\section{Extended spectrum $\beta$-lactamases}

133 Enterobacteriaceae isolates from 124 participants tested positive for ESBL production by double disc synergy. $71 \%$ of these were E.coli and 19\% K.pneumoniae. 99 (74.4\%) were PCR positive for at least one CTX-M enzyme, predominantly cluster 1 (64 isolates, 65\%) or 9 (32 isolates, 24\%). 3 isolates produced both cluster 1 and 9 CTX-M.

\section{Discussion}

Ceftriaxone resistance among clinical isolates of E.coli and K.pneumoniae from public hospitals in Singapore has reached $20-30 \%$ [15]. This incidence is rising and correlates with increasing prescription of broad spectrum antibiotics. We identified a similar high burden of ESBL-E colonization from rectal swab cultures. ESBL-E was detected in $29.4 \%$ of subjects who had been recently hospitalized and were recipients of antibiotics. $6.3 \%$ of subjects without healthcare contact or antibiotics were detected as ESBL-E colonized. The source of these organisms is of major concern.

In ESBL-E high prevalence countries such as Singapore, nosocomial transmission may not be the most important acquisition route. A study in a Swiss hospital with low ESBL-E prevalence observed infrequent transmission events even without contact isolation [16]. Modeling ESBL-E colonization in a French pediatric unit described the success of contact isolation in preventing nosocomial transmission, but its overall ineffectiveness due to the high incidence of sporadic cases from the community [17]. A similar finding from a study of ESBL-E transmission dynamics in Switzerland, detected more inpatient ESBL-E fecal carriage acquired from the community than in hospital [18]. Transmission between household members was also more frequent than between patients, though it was not clear if it was acquired from a common source - presumed to be food - or person-toperson.
The importance of recent antibiotic use in this study suggests that host susceptibility to colonization is a critical factor. Acquisition of ESBL-E from community or nosocomial sources may be facilitated by disrupting the host gut microbiome and providing a selective advantage for antibiotic-resistant organisms. Given the high rate of colonization in patients with no identified risk factors, an alternative hypothesis is that antibiotic use selects for ESBL-E from host indigenous gut flora, increasing the colonization burden. The sensitivity of rectal swabs for detection of ESBL-E has not been widely investigated, but was reported to correlate with density of colonization [19]. Antibiotics also generate de novo ESBL-E, by promoting DNA recombination events between gut bacteria [20].

Travel to Asia has been identified as a risk factor for ESBL-E fecal carriage in European and Australian prospective cohorts $[21,22]$. In both these studies, acquisition was associated with traveler's diarrhea, but not dependent on antibiotic use or healthcare contact. Curiously this is despite the isolation of ESBL-E from more than $90 \%$ of retail chicken meat in separate European studies $[23,24]$. This disconnect could be due to environmental or diet differences facilitating ESBL-E acquisition in Asia, or microbiome changes as a result of travel itself increasing susceptibility to colonization by antibioticresistant bacteria. CTX-M production by E.coli carried on chicken, pigs and cattle in China has been reported, and a systematic study of agricultural, food and clinical ESBL-E isolates in Asia would be interesting [25].

MRSA is a common healthcare-associated pathogen in Singapore. A colonization prevalence of $41 \%$ has been reported from nursing homes residents admitted to a public acute care hospital, and 6\% among all medical and surgical admissions [26]. Community-acquired MRSA strains are infrequently recognized as a cause of infections in Singapore, and its contribution to community MRSA colonization is not known [27].

Transmission of MRSA from colonized hands and environmental surfaces to new hosts is well established $[28,29]$. The high AOR in the logit model between 
hospitalization and MRSA colonization is consistent with this as a clinically significant factor. Antibiotic use has also been associated with colonization and infection by MRSA. Failure to identify this here may be due to the small number of cases [30,31]. Failure to isolate VRE was not unexpected, as the prevalence in Singapore is low outside of nosocomial outbreaks [32].

This study has a number of limitations. Accurate clinical data was dependent on recall by study participants or next-of-kin and was not independently verified by medical records. Data was not collected on antibiotic type and duration or frequency of prior contact with healthcare institutions as this was judged unreliable from patient recall. Our ability to analyze risk factors for colonization by MRSA and VRE was limited by the study design, due to the low detected prevalence. A case control study would help elaborate these.

Acquisition rates in hospital were not investigated, and this could have provided additional evidence to refine our conclusions. More accurate methods for detecting MRSA, ESBL-E and VRE colonization are now available: for example PCR-based detection methods may be more accurate and sensitive [33]. We were also unable to perform ESBL genotyping or bacterial strain typing, to determine if CTX-M-15 was the major enzyme in clusters 1 and 9, or if ESBL-E strains differed between apparent community- and hospital-acquired isolates. The prevalence of resistance mechanisms such as carbapenemases which have become of increasing concern was not assessed.

\section{Conclusions}

ESBL-E colonization was detected with high prevalence in subjects who were recently hospitalized and received antibiotics. Identifying subjects who were colonized by ESBL-E accurately was difficult using just the clinical data collected. Not least this was due to the significant proportion of ESBL-E associated with limited healthcare contact. Identifying the source of these organisms and the relative contributions of community and nosocomial transmission may be important for understanding how to control their spread.

\footnotetext{
Abbreviations

AOR: Adjusted odds ratio; Cl: Confidence interval; ED: Emergency department; ESBL-E: Extended-Spectrum $\beta$-lactamase-producing Enterobacteriaceae (ESBL-E); ESRF: End-stage renal failure; HCW: Healthcare worker; HIV: Human immunodeficiency virus; H-L: Hosmer Lemeshow; IAI: Intra-abdominal infection; OR: Odds ratio; PCR: Polymerase chain reaction; MRSA: Methicillin-resistant Staphylococcus aureus; VRE: Vancomycin-resistant Enterococcus.
}

\section{Competing interests}

The authors declare that they have no competing interests.

\section{Authors' contributions}

BY collected and analyzed data, and drafted the manuscript; PK conceived and designed the study, and performed culture and susceptibility testing; SC analyzed data and helped draft the manuscript; DL conceived and designed the study, collected and analyzed data and helped draft the manuscript; LY conceived and designed the study. All authors read and approved the final manuscript.

\section{Acknowledgments}

The study was funded by a Health Quality Improvement Fund grant from the Ministry of Health, Singapore. BY is supported by the National Healthcare Group Clinician Scientist Career Scheme.

\section{Author details}

'Institute of Infectious Diseases and Epidemiology, Communicable Diseases Centre, Singapore, Singapore. ${ }^{2}$ Department of Medicine, National University of Singapore, Singapore, Singapore. ${ }^{3}$ Department of Laboratory Medicine,

Tan Tock Seng Hospital, Singapore, Singapore. ${ }^{4}$ Department of Mathematics \& Statistics, La Trobe University, Melbourne, Australia.

Received: 19 September 2013 Accepted: 28 May 2014

Published: 2 June 2014

\section{References}

1. Molton JS, Tambyah PA, Ang BS, Ling ML, Fisher DA: The global spread of healthcare-associated multidrug-resistant bacteria: a perspective from Asia. Clin Infect Dis: an official publication of the Infectious Diseases Society of America 2013, 56(9):1310-1318.

2. Kumarasamy KK, Toleman MA, Walsh TR, Bagaria J, Butt F, Balakrishnan R, Chaudhary U, Doumith M, Giske CG, Irfan S, Krishnan P, Kumar AV, Maharjan S, Mushtaq S, Noorie T, Paterson DL, Pearson A, Perry C, Pike R, Rao B, Ray U, Sarma JB, Sharma M, Sheridan E, Thirunarayan MA, Turton J, Upadhyay S, Warner M, Welfare W, Livermore DM, et al: Emergence of a new antibiotic resistance mechanism in India, Pakistan, and the UK: a molecular, biological, and epidemiological study. Lancet Infect Dis 2010, 10(9):597-602.

3. Karim A, Poirel L, Nagarajan S, Nordmann P: Plasmid-mediated extendedspectrum beta-lactamase (CTX-M-3 like) from India and gene association with insertion sequence ISEcp1. FEMS Microbiol Lett 2001, 201(2):237-241.

4. Song JH, Hsueh PR, Chung DR, Ko KS, Kang Cl, Peck KR, Yeom JS, Kim SW, Chang HH, Kim YS, Jung SI, Son JS, So TM, Lalitha MK, Yang Y, Huang SG, Wang H, Lu Q, Carlos CC, Perera JA, Chiu CH, Liu JW, Chongthaleong A, Thamlikitkul V, Van PH, ANSORP Study Group: Spread of methicillinresistant Staphylococcus aureus between the community and the hospitals in Asian countries: an ANSORP study. J Antimicrob Chemother 2011, 66(5):1061-1069.

5. Lu PL, Liu YC, Toh HS, Lee YL, Liu YM, Ho CM, Huang CC, Liu CE, Ko WC, Wang JH, Tang HJ, Yu KW, Chen YS, Chuang YC, Xu Y, Ni Y, Chen YH, Hsueh PR: Epidemiology and antimicrobial susceptibility profiles of Gramnegative bacteria causing urinary tract infections in the Asia-Pacific region: 2009-2010 results from the Study for Monitoring Antimicrobial Resistance Trends (SMART). Int J Antimicrob Agents 2012, 40(Suppl):S37-S43.

6. Sheng WH, Badal RE, Hsueh PR: Distribution of extended-spectrum betalactamases, AmpC beta-lactamases, and carbapenemases among Enterobacteriaceae isolates causing intra-abdominal infections in the Asia-Pacific region: results of the study for Monitoring Antimicrobial Resistance Trends (SMART). Antimicrob Agents Chemother 2013, 57(7):2981-2988

7. Luvsansharav UO, Hirai I, Niki M, Nakata A, Yoshinaga A, Moriyama T, Yamamoto Y: Prevalence of fecal carriage of extended-spectrum betalactamase-producing Enterobacteriaceae among healthy adult people in Japan. J Infect Chemotherapy: official journal of the Japan Society of Chemotherapy 2011, 17(5):722-725.

8. Sasaki T, Hirai I, Niki M, Nakamura T, Komalamisra C, Maipanich W, Kusolsuk T, Sa-Nguankiat S, Pubampen S, Yamamoto Y: High prevalence of CTX-M beta-lactamase-producing Enterobacteriaceae in stool specimens obtained from healthy individuals in Thailand. J Antimicrob Chemother 2010, 65(4):666-668.

9. Reddy P, Malczynski M, Obias A, Reiner S, Jin N, Huang J, Noskin GA Zembower T: Screening for extended-spectrum beta-lactamaseproducing Enterobacteriaceae among high-risk patients and rates of subsequent bacteremia. Clin Infect Dis: an official publication of the Infectious Diseases Society of America 2007, 45(7):846-852.

10. Davis KA, Stewart JJ, Crouch HK, Florez CE, Hospenthal DR: Methicillinresistant Staphylococcus aureus (MRSA) nares colonization at hospital admission and its effect on subsequent MRSA infection. Clin Infect Dis: an 
official publication of the Infectious Diseases Society of America 2004, 39(6):776-782

11. Schwaber MJ, Carmeli Y: Mortality and delay in effective therapy associated with extended-spectrum beta-lactamase production in Enterobacteriaceae bacteraemia: a systematic review and meta-analysis. J Antimicrob Chemother 2007, 60(5):913-920.

12. Lodise TP, McKinnon PS, Swiderski L, Rybak MJ: Outcomes analysis of delayed antibiotic treatment for hospital-acquired Staphylococcus aureus bacteremia. Clin Infect Dis: an official publication of the Infectious Diseases Society of America 2003, 36(11):1418-1423.

13. Valverde A, Coque TM, Sanchez-Moreno MP, Rollan A, Baquero F, Canton R: Dramatic increase in prevalence of fecal carriage of extended-spectrum beta-lactamase-producing Enterobacteriaceae during nonoutbreak situations in Spain. J Clin Microbiol 2004, 42(10):4769-4775.

14. CLSI: Performance Standards for Antimicrobial Susceptibility Testing; Seventeenth Informational Supplement. CLSI document M100-S17. Pennsylvania, USA: Clinical and Laboratory Standards Institute; 2007.

15. Hsu LY, Tan TY, Tam VH, Kwa A, Fisher DA, Koh TH: Surveillance and correlation of antibiotic prescription and resistance of Gram-negative bacteria in Singaporean hospitals. Antimicrob Agents Chemother 2010, 54(3):1173-1178

16. Tschudin-Sutter S, Frei R, Dangel M, Stranden A, Widmer AF: Rate of transmission of extended-spectrum beta-lactamase-producing enterobacteriaceae without contact isolation. Clin Infect Dis: an official publication of the Infectious Diseases Society of America 2012, 55(11):1505-1511.

17. Domenech de Celles M, Zahar JR, Abadie V, Guillemot D: Limits of patient isolation measures to control extended-spectrum beta-lactamaseproducing Enterobacteriaceae: model-based analysis of clinical data in a pediatric ward. BMC Infect Dis 2013, 13:187.

18. Hilty M, Betsch BY, Bogli-Stuber K, Heiniger N, Stadler M, Kuffer M, Kronenberg A, Rohrer C, Aebi S, Endimiani A, Droz S, Mühlemann K: Transmission dynamics of extended-spectrum beta-lactamase-producing Enterobacteriaceae in the tertiary care hospital and the household setting. Clin Infect Dis: an official publication of the Infectious Diseases Society of America 2012, 55(7):967-975.

19. Lautenbach E, Harris AD, Perencevich EN, Nachamkin I, Tolomeo P, Metlay JP: Test characteristics of perirectal and rectal swab compared to stool sample for detection of fluoroquinolone-resistant Escherichia coli in the gastrointestinal tract. Antimicrob Agents Chemother 2005, 49(2):798-800.

20. Modi SR, Lee HH, Spina CS, Collins JJ: Antibiotic treatment expands the resistance reservoir and ecological network of the phage metagenome. Nature 2013, 499(7457):219-222.

21. Tangden T, Cars O, Melhus A, Lowdin E: Foreign travel is a major risk factor for colonization with Escherichia coli producing CTX-M-type extended-spectrum beta-lactamases: a prospective study with Swedish volunteers. Antimicrob Agents Chemother 2010, 54(9):3564-3568

22. Kennedy K, Collignon P: Colonisation with Escherichia coli resistant to "critically important" antibiotics: a high risk for international travellers Eur J Clin Microbiol Infect Dis: official publication of the European Society of Clinical Microbiology 2010, 29(12):1501-1506.

23. Egea P, Lopez-Cerero L, Torres E, Gomez-Sanchez Mdel C, Serrano L, Navarro Sanchez-Ortiz MD, Rodriguez-Bano J, Pascual A: Increased raw poultry meat colonization by extended spectrum beta-lactamase-producing Escherichia coli in the south of Spain. Int J Food Microbiol 2012, 159(2):69-73.

24. Leverstein-van Hall MA, Dierikx CM, Cohen Stuart J, Voets GM, van den Munckhof MP, van Essen-Zandbergen A, Platteel T, Fluit AC, van de SandeBruinsma N, Scharinga J, Bonten MJ, Mevius DJ, National ESBL surveillance group: Dutch patients, retail chicken meat and poultry share the same ESBL genes, plasmids and strains. Clin Microbiol Infect: the official publication of the European Society of Clinical Microbiology and Infectious Diseases 2011, 17(6):873-880

25. Zheng H, Zeng Z, Chen S, Liu Y, Yao Q, Deng Y, Chen X, Lv L, Zhuo C, Chen Z, Liu JH: Prevalence and characterisation of CTX-M beta-lactamases amongst Escherichia coli isolates from healthy food animals in China. Int J Antimicrob Agents 2012, 39(4):305-310.

26. Verrall A, Merchant R, Dillon J, Ying D, Fisher D: Impact of nursing home residence on hospital epidemiology of meticillin-resistant Staphylococcus aureus: a perspective from Asia. J Hosp Infect 2013, 83(3):250-252.

27. Hsu LY, Koh YL, Chlebicka NL, Tan TY, Krishnan P, Lin RT, Tee N, Barkham T, Koh TH: Establishment of ST30 as the predominant clonal type among community-associated methicillin-resistant Staphylococcus aureus isolates in Singapore. J Clin Microbiol 2006, 44(3):1090-1093.

28. Coello R, Jimenez J, Garcia M, Arroyo P, Minguez D, Fernandez C, Cruzet F, Gaspar C: Prospective study of infection, colonization and carriage of methicillin-resistant Staphylococcus aureus in an outbreak affecting 990 patients. Eur J Clin Microbiol Infect Dis: official publication of the European Society of Clinical Microbiology 1994, 13(1):74-81.

29. Boyce JM, Potter-Bynoe G, Chenevert C, King T: Environmental contamination due to methicillin-resistant Staphylococcus aureus: possible infection control implications. Infect Control Hosp Epidemiol: the official journal of the Society of Hospital Epidemiologists of America 1997, 18(9):622-627.

30. Hill DA, Herford T, Parratt D: Antibiotic usage and methicillin-resistant Staphylococcus aureus: an analysis of causality. J Antimicrob Chemother 1998, 42(5):676-677.

31. Graffunder EM, Venezia RA: Risk factors associated with nosocomial methicillin-resistant Staphylococcus aureus (MRSA) infection including previous use of antimicrobials. J Antimicrob Chemother 2002, 49(6):999-1005.

32. Cai Y, Chan JP, Fisher DA, Hsu LY, Koh TH, Krishnan P, Kwa AL, Tan TY, Tee NW: Vancomycin-resistant Enterococci in Singaporean hospitals: 5-year results of a multi-centre surveillance programme. Ann Acad Med Singap 2012, 41(2):77-81.

33. Win MK, Yung CF, Poh BF, Krishnan PU, Seet SK, Leo YS, Ang B: Evaluation of universal methicillin-resistant Staphylococcus aureus screening using nasal polymerase chain reaction compared with nasal, axilla, and groin and throat and perianal cultures in a hospital setting. Infect Control Hosp Epidemiol: the official journal of the Society of Hospital Epidemiologists of America 2013, 34(12):1335-1337.

doi:10.1186/1471-2334-14-298

Cite this article as: Young et al:: A prospective observational study of the prevalence and risk factors for colonization by antibiotic resistant bacteria in patients at admission to hospital in Singapore. BMC Infectious Diseases 2014 14:298.

\section{Submit your next manuscript to BioMed Central and take full advantage of:}

- Convenient online submission

- Thorough peer review

- No space constraints or color figure charges

- Immediate publication on acceptance

- Inclusion in PubMed, CAS, Scopus and Google Scholar

- Research which is freely available for redistribution 